\title{
One-Year Comparative Evaluation of iStent or iStent inject Implantation Combined with Cataract Surgery in a Single Center
}

\author{
Ricardo Augusto Paletta Guedes • Daniela Marcelo Gravina • \\ Jonathan Clive Lake · Vanessa Maria Paletta Guedes · Alfredo Chaoubah
}

Received: July 25, 2019 / Published online: August 22, 2019

(C) The Author(s) 2019

\section{ABSTRACT}

Introduction: This retrospective consecutive case series assessed 12-month effectiveness and safety of iStent ${ }^{\circledR}$ or iStent inject ${ }^{\circledR}$ trabecular micro-bypass implants with cataract surgery in patients with open-angle glaucoma (OAG) in a real-world clinical setting.

Methods: Effectiveness outcomes consisted of intraocular pressure (IOP) reduction; glaucoma medication reduction; proportions of eyes achieving IOP $<18,<15$, or $<12 \mathrm{mmHg}$; and proportional analysis of medication usage. Safety outcomes included adverse events, secondary surgeries, and best-corrected visual acuity (BCVA).

Results: This evaluation included 58 eyes with OAG (35 iStent, 23 iStent inject), with $96.6 \%$ of eyes having mild or moderate glaucoma.

Enhanced Digital Features To view enhanced digital features for this article go to https://doi.org/10.6084/ m9.figshare.9577487.

R. A. P. Guedes $(\bowtie) \cdot$ D. M. Gravina

V. M. P. Guedes

Paletta Guedes Eye Institute, Juiz de Fora,

Minas Gerais, Brazil

e-mail: palettaguedes@yahoo.com

R. A. P. Guedes · A. Chaoubah

Federal University of Juiz de Fora, Juiz de Fora,

Minas Gerais, Brazil

J. C. Lake

Brasilia Vision Hospital, Brasilia, Brazil
Diagnoses included primary open-angle glaucoma (the majority; 72.4\%), pseudoexfoliative glaucoma, and pigmentary glaucoma. Baseline mean IOP and medications were statistically comparable between groups: $16.1 \pm 3.6 \mathrm{mmHg}$ on a mean of $1.8 \pm 0.8$ medications in the iStent group, and $16.2 \pm 3.1 \mathrm{mmHg}$ on a mean of $1.7 \pm 0.8$ medications in the iStent inject group. Twelve months after stent-cataract surgery, mean IOP was significantly lower in the iStent inject group than in the iStent group $(13.1 \mathrm{mmHg}$ vs. $15.4 \mathrm{mmHg}$, respectively; $p<0.001$ ), and the percent reduction in IOP from baseline was significantly greater in iStent inject eyes than in iStent eyes $(19.1 \%$ vs. $4.3 \%$ reduction, respectively; $\quad p<0.001)$. At 12 months postoperative, significantly greater proportions of iStent inject eyes than iStent eyes achieved IOP $<18 \mathrm{mmHg}(100 \%$ vs. $80.0 \%$ of eyes, respectively; $p=0.035$ ), IOP $<15 \mathrm{mmHg}$ $(73.9 \%$ vs. $34.3 \%$ of eyes, respectively; $p=0.003)$, and IOP $<12 \mathrm{mmHg}(26.1 \%$ vs. $0 \%$ of eyes, respectively; $p=0.002$ ). Meanwhile, both groups achieved significant medication reductions at 12 months vs. baseline $(94.1 \%$ reduction in iStent inject eyes, $p<0.0001$; and $72.2 \%$ reduction in iStent eyes, $p<0.0001$ ), with the percent reduction being significantly greater in iStent inject eyes than in iStent eyes $(p=0.023)$. At 12 months, mean number of medications was significantly lower in iStent inject eyes than iStent eyes ( 0.1 vs. 0.5 medications, respectively; $p=0.021$ ), and significantly 
more iStent inject eyes (95.7\%) than iStent eyes $(71.4 \%)$ were off medications entirely $(p=0.021)$. A similarly high safety profile was observed in both groups.

Conclusion: iStent or iStent inject implantation with cataract surgery resulted in substantial and safe reductions in IOP and medications through 12 months postoperative. Consistent with prior observations, greater efficacy was observed with iStent inject than with iStent.

Funding: The Rapid Service Fees were funded by Glaukos Corporation.

Keywords: Cataract; Glaucoma; Intraocular pressure; iStent; iStent inject; Medication; Microinvasive glaucoma surgery (MIGS); Safety; Second-generation; Trabecular microbypass

\section{INTRODUCTION}

Already the top cause of permanent blindness globally, glaucoma is expected to increase even further in prevalence in coming decades [1]. Currently, the central objective of all glaucoma treatments is to reduce intraocular pressure (IOP), which is the primary risk factor for the development and progression of glaucoma and visual field loss. Several foundational landmark studies soundly established the strong protective connection between lowering IOP and reducing glaucoma damage [2-5]. One of the studies, the Early Manifest Glaucoma Trial (EMGT), even assigned a numerical measure to this reduced risk: approximately 10\% reduction in the risk of glaucoma progression for every $1 \mathrm{mmHg}$ of IOP reduction [3].

A number of medical and surgical therapies are available to treat glaucoma, each of which has benefits and downsides. Conservative interventions typically include topical medications and/or laser trabeculoplasty procedures $[6,7]$. Medications are reliably effective in reducing IOP and are usually the first-line intervention, but they are associated with side effects, ocular surface deterioration, costs, inconsistency with drop instillation, and regrettably low treatment adherence [8-12]. Laser procedures also are an effective early intervention, but their effects are known to decline over time, thereby posing a recurring challenge for patients with this lifelong disease [13]. Traditional incisional glaucoma surgeries such as trabeculectomy or tube implantation can produce dramatic IOP reduction, but they also carry a risk profile that includes sightthreatening complications such as hypotony maculopathy, choroidal detachment and effusion, endophthalmitis, anterior chamber collapse, and bleb-related infections or inflammation [14-16].

Micro-invasive glaucoma surgery (MIGS) has been developed and refined over the past decade to bridge the gap between medication and filtering surgeries [17]. The first Food and Drug Administration (FDA)-approved MIGS device, the iStent trabecular micro-bypass, as well as the more recently introduced iStent inject trabecular micro-bypass (Fig. 1), have been examined in a wide range of studies and clinical settings. The devices have been studied with and without cataract surgery, in various severities of glaucoma, in different types of glaucoma (e.g., primary open-angle glaucoma, POAG; pseudoexfoliative glaucoma, PXG; normal-tension glaucoma, NTG; combined-mechanism glaucoma, CMG) as well as in ocular hypertension [18-40]. The data have consistently shown favorable safety together with substantial and durable reductions in IOP and medications.

The present cohort includes consecutive cases of either the iStent (comprising one firstgeneration stent) or iStent inject (comprising two second-generation stents) trabecular microbypass implanted with concomitant cataract surgery. Both devices decrease IOP by creating a patent pathway (or pathways) through the trabecular meshwork into Schlemm's canal, thereby promoting egress of aqueous humor from the anterior chamber. The cohort's inclusion of both devices allows us to distinguish the effects of two stents versus one (iStent inject versus iStent, respectively), as well as to assess for any potential benefit of the design features of the newer iStent inject device. The current report draws data only from patients having 12 months of follow-up visits (consistent cohort, $n=58$ ). A prior publication [40] showed outcomes for patients having 6 months of 


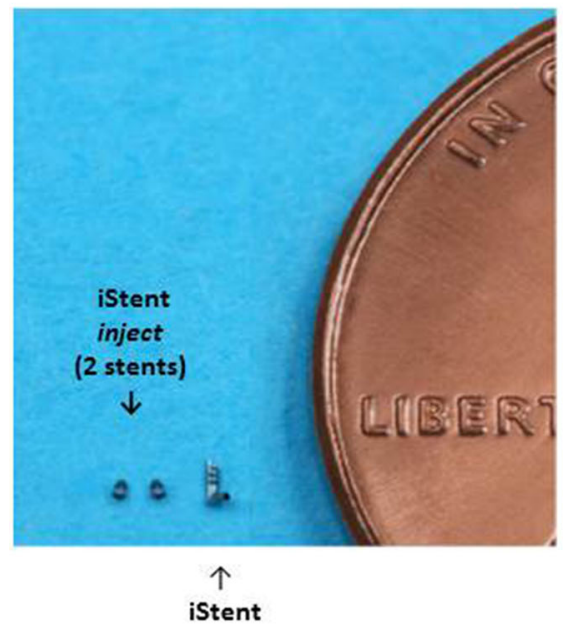

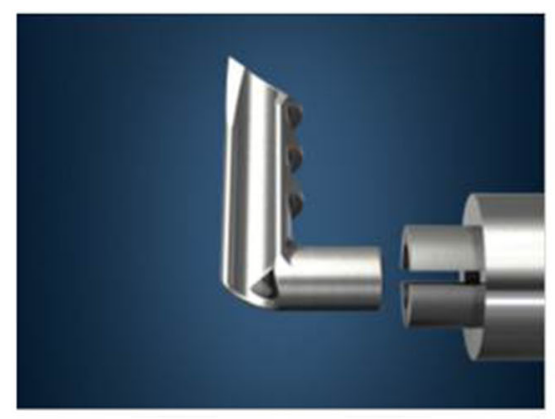

iStent $^{2}$ (1 stent per device)

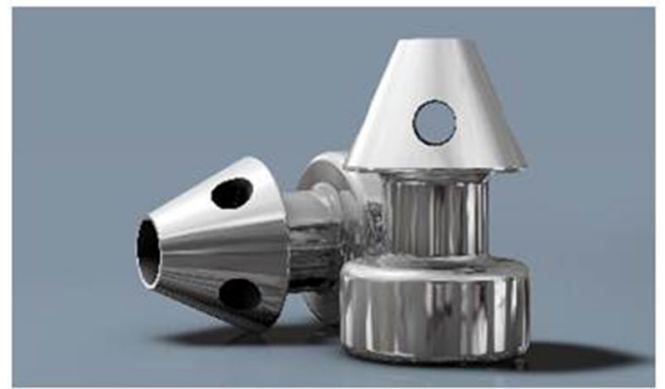

iStent inject $^{2}$

(2 stents per device, each with 4 lateral outlet lumens for multidirectional outflow)

Fig. 1 iStent ${ }^{\circledR}$ and iStent inject ${ }^{\circledR}$ trabecular micro-bypass stents, with relative dimensions (image courtesy of Glaukos Corp., San Clemente, CA, USA)

follow-up visits $(n=73)$. Given that each report pertains to a consistent group of patients at a given point in time ( 6 months or 12 months), the 6- and 12-month analyses are not directly comparable.

The aim of the present study was to compare the 12-month performance and safety outcomes of iStent and iStent inject implantation in combination with cataract surgery in eyes with mild to moderate open-angle glaucoma. This report contributes one of the first datasets with side-by-side data on the two devices in a realworld clinical population.

\section{METHODS}

In this longitudinal retrospective study, we evaluated 12-month outcomes of consecutive eyes that underwent implantation of either iStent or iStent inject in combination with cataract surgery from June 2017 to June 2018 . The choice between implants was based predominantly on the availability of the different implants on the Brazilian market. To eliminate any possible impact of a learning curve on outcomes, we excluded the first 10 iStent cases and the first 5 iStent inject cases from the cohort. All surgeries were performed in a single private practice ophthalmic surgery center by one surgeon (RG).

Inclusion criteria included age over 18 years, diagnosis of OAG, follow-up data through 12 months postoperative, cataract requiring surgery, and the need to reduce IOP or medications. Eyes were required to have anterior chamber angle grade of III or IV (per the modified Shaffer classification), where at least the scleral spur was visible under gonioscopic view. Preoperative glaucoma severity was classified using the Hodapp-Parrish-Anderson visual field criteria (mild, mean deviation (MD) no worse 
than $-6 \mathrm{~dB}$; moderate, MD worse than $-6 \mathrm{~dB}$ but no worse than $-12 \mathrm{~dB}$; severe, MD worse than $-12 \mathrm{~dB}$ ) [41]. We excluded eyes with shorter follow-up than 12 months.

Main outcome measures through 12 months included mean IOP and medications; percentage reduction in IOP and medications versus baseline; proportions of eyes with IOP $<18 \mathrm{mmHg}$, $<15 \mathrm{mmHg}$, or $<12 \mathrm{mmHg}$; and proportional analysis of medication usage. IOP was measured by Goldmann applanation. Safety assessments included intraoperative and postoperative adverse events, secondary surgeries, and best-corrected visual acuity (BCVA, Snellen). Gonioscopy was performed preoperatively and at every postoperative visit during follow-up in order to evaluate the angle and stent positioning.

We used the chi-square test and Student's $t$ test, respectively, for the analysis of categorical and numerical variables. Statistical analysis was performed in SPSS. We considered a $p$ value of 0.05 for statistical significance. All procedures were in accordance with the Institutional Review Board of the Paletta Guedes Eye Institute and with the 1964 Helsinki declaration and its later amendments or comparable ethical standards. Formal trial registration was not required for this retrospective analysis, given that it included only patients from the surgeon's real clinical practice who already received treatment.

\section{RESULTS}

All eyes underwent uncomplicated phacoemulsification cataract surgery, followed by implantation of either the iStent $(n=35)$ or the iStent inject $(n=23)$ trabecular micro-bypass. Preoperative demographics and ocular parameters are displayed in Table 1. Almost all eyes (96.6\%) had mild or moderate glaucoma, and the predominant diagnosis was primary open-angle glaucoma (POAG, $72.4 \%$ of eyes), with the remaining eyes having pseudoexfoliative glaucoma (PXG) or pigmentary glaucoma (PG). At baseline, the groups were statistically comparable in nearly all parameters, including IOP, medications, and glaucoma stage. Baseline age
Table 1 Baseline demographic and ocular characteristics

\begin{tabular}{|c|c|c|c|}
\hline Characteristics & $\begin{array}{l}\text { iStent }+ \\
\text { cataract } \\
\text { surgery } \\
n=35\end{array}$ & $\begin{array}{l}\text { iStent } \text { inject } \\
+ \text { cataract } \\
\text { surgery } \\
n=23\end{array}$ & $p$ value \\
\hline $\begin{array}{l}\text { Age } \\
\quad(\text { mean } \pm S D), \\
\text { years }\end{array}$ & $67.8 \pm 8.9$ & $73.4 \pm 7.4$ & $0.013^{\mathrm{a}}$ \\
\hline $\begin{array}{l}\text { Baseline IOP, } \\
\text { mmHg }\end{array}$ & $16.1 \pm 3.6$ & $16.2 \pm 3.1$ & $0.882^{\mathrm{a}}$ \\
\hline $\begin{array}{l}\text { Baseline number } \\
\text { of medications }\end{array}$ & $1.8 \pm 0.8$ & $1.7 \pm 0.8$ & $0.565^{\mathrm{a}}$ \\
\hline \multicolumn{4}{|l|}{ Race } \\
\hline Caucasian & $74.3 \%$ & $91.3 \%$ & $0.203^{\mathrm{b}}$ \\
\hline African descent & $25.7 \%$ & $8.7 \%$ & \\
\hline \multicolumn{4}{|l|}{ Gender } \\
\hline Male & $17.1 \%$ & $43.5 \%$ & $0.038^{\mathrm{b}}$ \\
\hline Female & $82.9 \%$ & $56.5 \%$ & \\
\hline \multicolumn{4}{|l|}{ Glaucoma stage } \\
\hline Mild & $85.7 \%$ & $82.6 \%$ & $0.181^{b}$ \\
\hline Moderate & $14.3 \%$ & $8.7 \%$ & \\
\hline Advanced & $0.0 \%$ & $8.7 \%$ & \\
\hline \multicolumn{4}{|l|}{ Laterality } \\
\hline OD & $48.6 \%$ & $47.8 \%$ & $0.584^{\mathrm{b}}$ \\
\hline OS & $51.4 \%$ & $52.2 \%$ & \\
\hline \multicolumn{4}{|c|}{ Baseline visual acuity } \\
\hline $20 / 30$ or better & $51.4 \%$ & $52.2 \%$ & $0.338^{\mathrm{b}}$ \\
\hline $\begin{array}{l}20 / 40 \text { to } \\
20 / 100\end{array}$ & $40.0 \%$ & $47.8 \%$ & \\
\hline $20 / 200$ or worse & $8.6 \%$ & $0.0 \%$ & \\
\hline
\end{tabular}

was slightly higher in iStent inject eyes, and there were slightly more women in the iStent group. 


\section{Intraocular Pressure}

Figure 2 shows the preoperative and 12-month mean IOP for the iStent and iStent inject groups. In iStent eyes, mean IOP reduced from $16.1 \pm 3.6 \mathrm{mmHg}$ at baseline to $15.4 \pm 2.4 \mathrm{mmHg}$ at 12 months postoperative $(p=0.201)$. In iStent inject eyes, mean IOP reduced from $16.2 \pm 3.1 \mathrm{mmHg}$ at baseline to $13.1 \pm 2.2 \mathrm{mmHg}$ at 12 months postoperative $(p<0.001)$. Mean percent IOP reduction was significantly greater in iStent inject eyes (19.1\%) than iStent eyes $(4.3 \%) \quad(p<0.001)$. Even though baseline IOP was similar in the two groups $(p=0.882)$, mean IOP at 12 months was significantly lower in the iStent inject group than the iStent group $(p<0.001)$.

Figure 3 depicts the distribution of IOP at 12 months postoperative in each group, which showed a lower IOP range for iStent inject than iStent eyes. Correspondingly, a significantly greater proportion of iStent inject eyes than iStent eyes achieved IOP $<18 \mathrm{mmHg}(100 \%$ vs.
$80.0 \%$ of eyes, respectively; $p=0.035$ ), IOP $<15 \mathrm{mmHg} \quad(73.9 \%$ vs. $34.3 \%$ of eyes, respectively; $p=0.003$ ), and IOP $<12 \mathrm{mmHg}$ (26.1\% vs. $0 \%$ of eyes, respectively; $p=0.002$ ) at 12 months (Fig. 4).

\section{Medications}

Figure 5 shows the mean number of medications from baseline to 12 months for each group. At baseline, the iStent and iStent inject groups had a similar mean number of medications (1.8 vs. $1.7, p=0.565)$. At 12 months postoperative, iStent inject eyes reduced medication burden by $94.1 \%(p<0.0001)$ and iStent eyes decreased their medication burden by $72.2 \%(p<0.0001)$. The percent reduction in medications was significantly greater in iStent inject eyes than in iStent eyes $(p=0.023)$. Additionally, iStent inject eyes had a significantly lower mean number of medications than iStent eyes at 12 months $(0.1 \pm 0.2$ medications

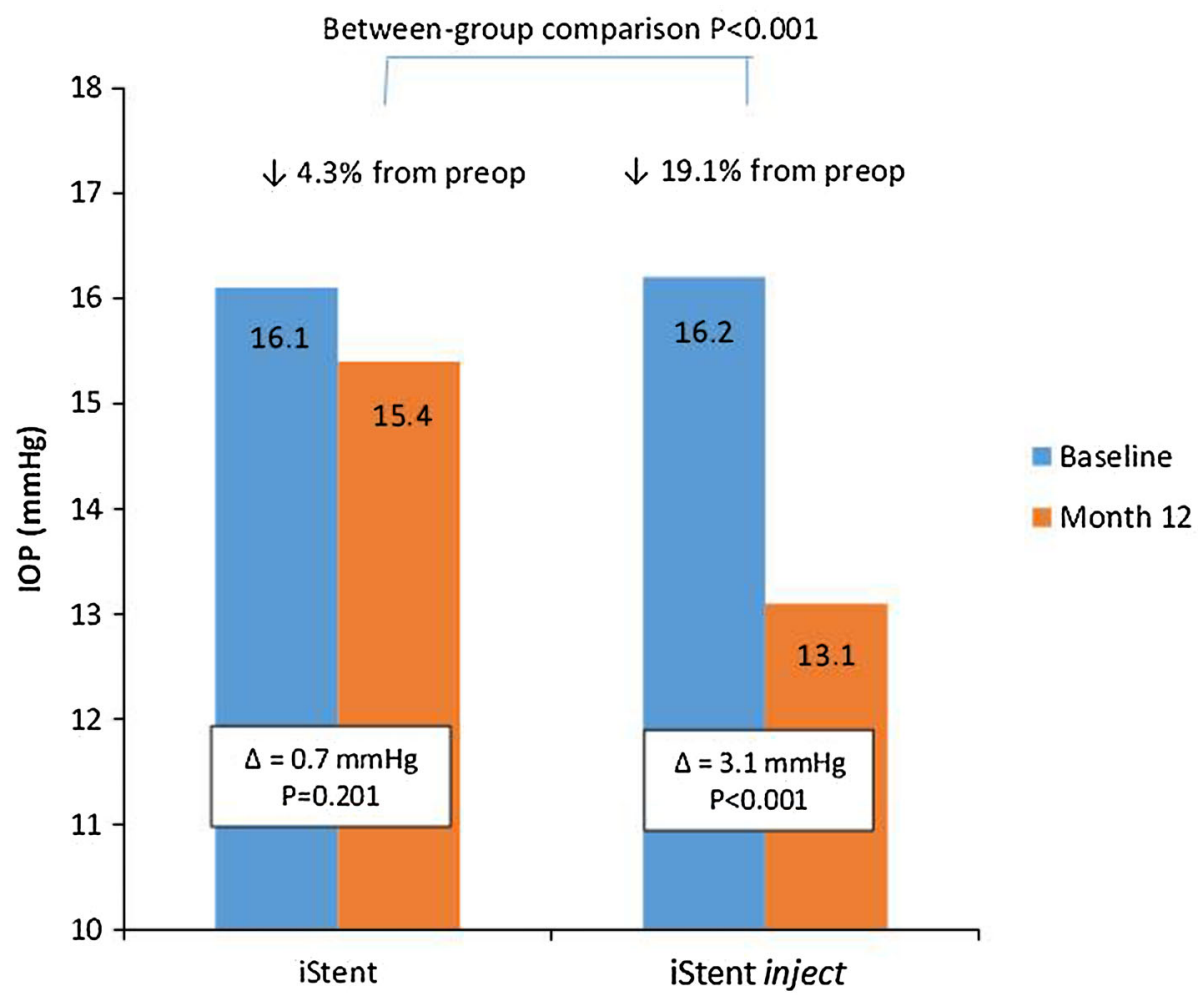

Fig. 2 Baseline and month 12 mean intraocular pressure 


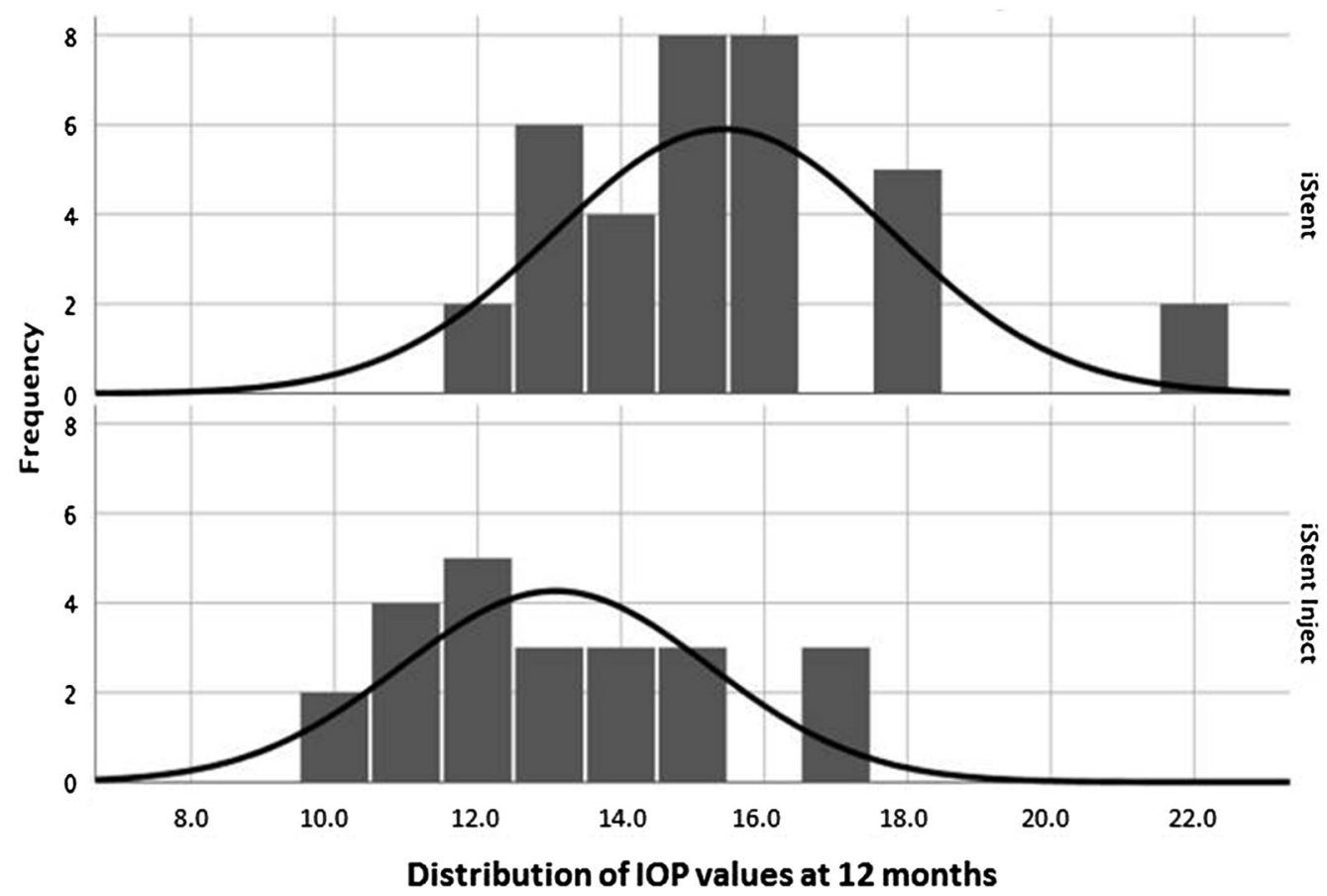

Fig. 3 Intraocular pressure distribution at 12 months postoperative

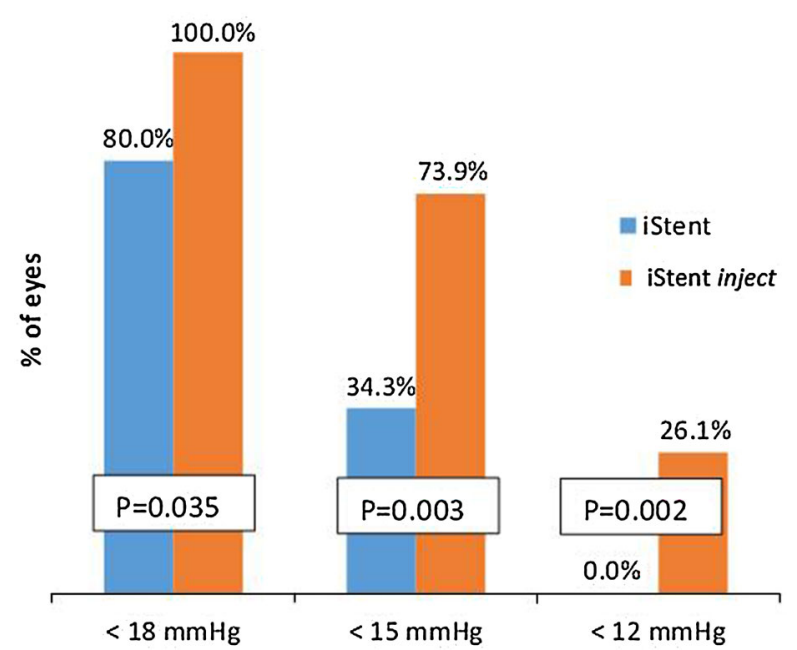

Fig. 4 Proportional analysis of intraocular pressure at 12 months postoperative

vs. $\quad 0.5 \pm 0.8$ medications, respectively; $p=0.021)$.

Figures 6 and 7 show the distributions of medication burden for each group at baseline and 12 months, respectively. At 12 months postoperative, both groups substantially decreased the percentage of eyes on two or more medications and increased the percentage of eyes on zero medications. This shift toward lower medication burden was particularly notable in the iStent inject group. Prior to surgery, $62.9 \%$ of iStent eyes and $52.1 \%$ of iStent inject eyes were on two or more medications; meanwhile, $2.9 \%$ and $4.3 \%$ of eyes, respectively, were medication-free. Twelve months after surgery, only $14.3 \%$ of iStent eyes vs. $0 \%$ of iStent inject eyes were on two or more medications $(p=0.057)$, and $71.4 \%$ of iStent eyes vs. $95.7 \%$ of iStent inject eyes were medication-free $(p=0.021)$. The reductions in number of medications versus baseline were statistically significant at all time points $(p<0.001)$ for both groups.

\section{Safety}

Both groups demonstrated a favorable safety profile. A similarly high proportion of patients $(87-89 \%)$ in each group had no intraoperative adverse events (between-group comparison, $p=0.336)$. There were two intraoperative cases 


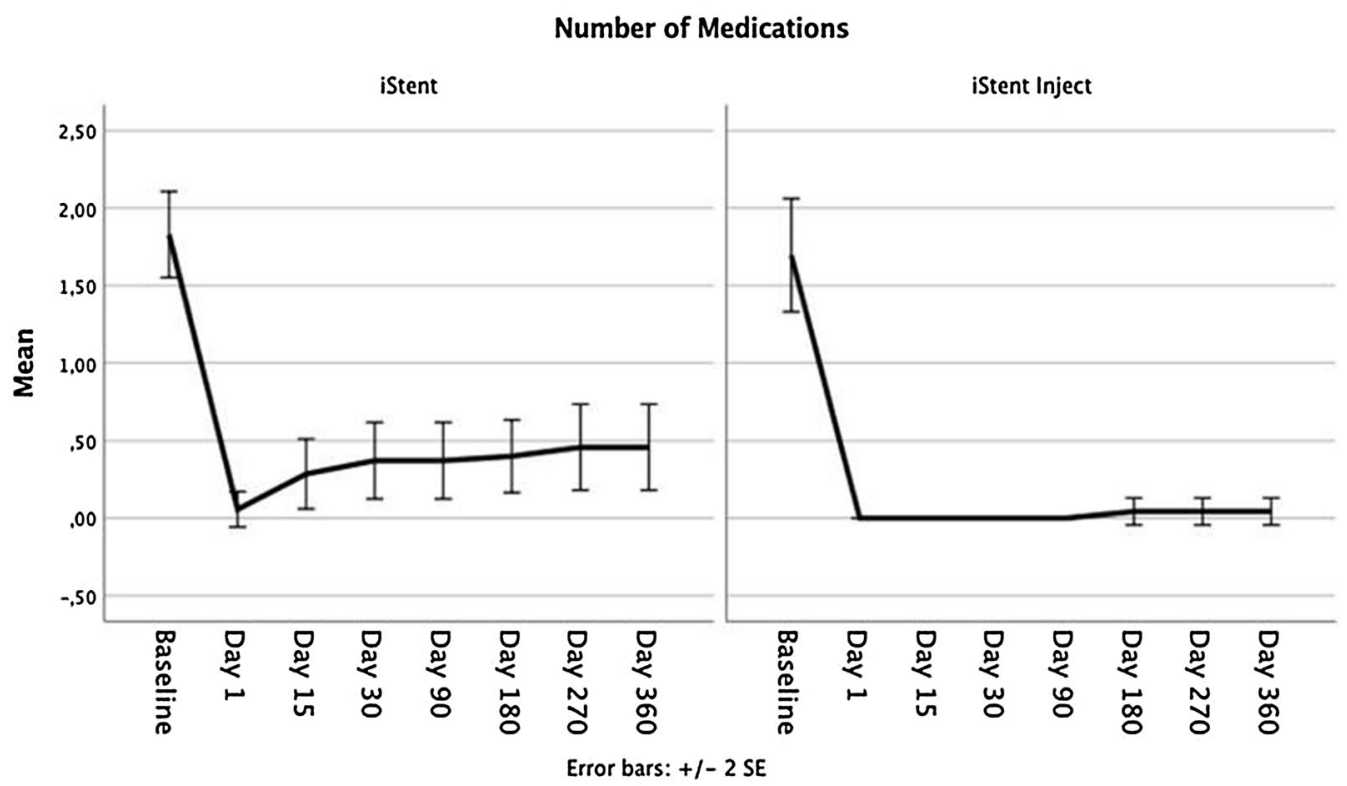

Fig. 5 Mean number of medications from baseline to 12 months postoperative

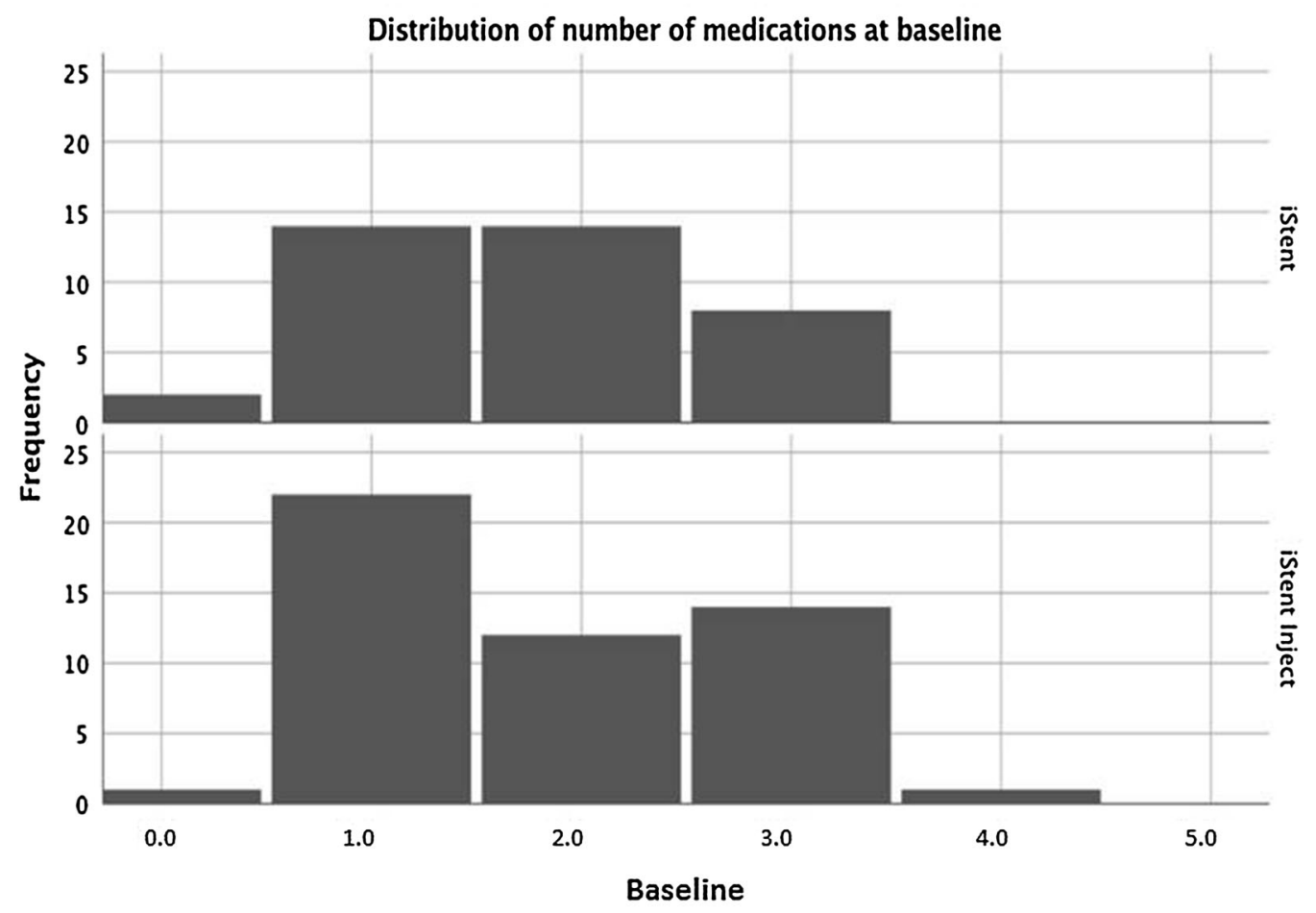

Fig. 6 Baseline distribution of number of medications

of blood reflux in the iStent group that resulted in no associated complications or sequelae. There were two cases of stent misplacement (under- or over-implantation) in iStent eyes and three cases in iStent inject eyes, as identified by intraoperative and postoperative gonioscopy. 


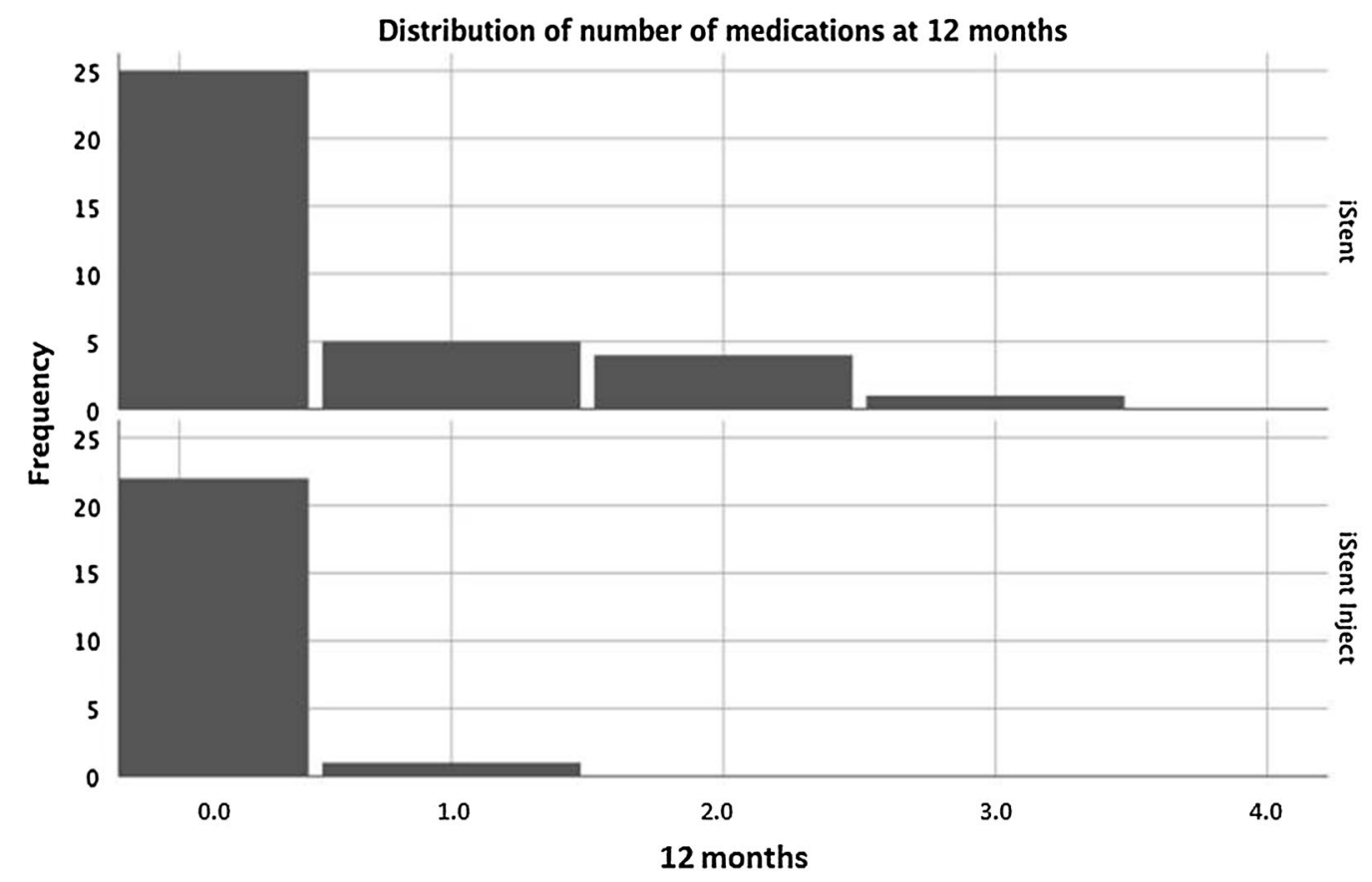

Fig. 7 Month 12 distribution of number of medications

These cases resulted in no sequelae or IOP ramifications, and no additional treatment measures were taken.

During 12 months of follow-up, no adverse events and no secondary glaucoma surgeries occurred in the iStent inject group. In the iStent group, one eye had focal peripheral anterior synechiae (PAS) occluding the internal ostia of the iStent at 3 months postoperative. The occlusion was corrected uneventfully with Nd:YAG laser iridotomy and resulted in no sequelae. One iStent eye underwent secondary glaucoma surgery postoperatively (non-penetrating deep sclerectomy, NPDS). This eye had elevated IOP due to systemic steroid use for asthma; no associated stent malfunction was noted. NPDS was completed at 3 months postoperative, and last reported IOP was $14 \mathrm{mmHg}$ on zero medications. No other postoperative adverse events or secondary surgeries occurred throughout follow-up.

Consistent with expectations for phacoemulsification cataract surgery, visual acuity improved in both groups versus baseline. The proportion of iStent eyes with visual acuity of $20 / 30$ or better increased from $51.4 \%$ preoperatively to $82.9 \%$ at 12 months postoperative
( $p=0.001)$; and the proportion of iStent inject eyes with 20/30 or better visual acuity increased from $52.2 \%$ preoperatively to $95.7 \%$ at 12 months postoperative $(p<0.001)$. There were no statistical differences between groups concerning visual acuity proportions at baseline $(p=0.338)$ or at 12 months $(p=0.147)$.

\section{DISCUSSION}

This report constitutes one of the first longitudinal comparative datasets of iStent and iStent inject with cataract surgery in eyes with glaucoma. In a real-world, single-site, single-surgeon setting, this 12-month series revealed substantial reductions in IOP and medications, with consistently higher efficacy outcomes after iStent inject than iStent. These improvements in IOP and medications were accompanied by excellent safety. Given that the dataset included consecutive all-comers in the surgeon's actual patient population, the outcomes may be more generalizable to the broader ophthalmic community of physicians and patients deciding upon glaucoma treatment. 
The IOP reduction achieved after iStent inject implantation was significantly greater than that of iStent implantation. Trabecular devices act upon the physiologic trabecular outflow pathway and do not eliminate the component of IOP contributed by episcleral venous pressure. As a result, trabecular devices traditionally have been thought to be limited in the level of postoperative IOP they can achieve. Postsurgical IOP in the iStent group was consistent with this expectation, with IOP reducing from $16.1 \mathrm{mmHg}$ at baseline to $15.4 \mathrm{mmHg}$ at 12 months. Meanwhile, iStent inject resulted in significantly lower final IOP $(13.1 \mathrm{mmHg})$, suggesting that iStent inject may be able to surpass the IOP limitations traditionally ascribed to trabecular devices. Within IDE (investigational device exemption) pivotal trials, iStent inject has shown the lowest postoperative IOP $(17.1 \mathrm{mmHg})$ of all trabecular micro-bypass devices [18, 31, 42]. The outcomes in the present study suggest that in real-world usage, postoperative IOP with iStent inject could be even lower. Correspondingly, significantly greater proportions of iStent inject eyes reached the IOP outcomes $(<18 \mathrm{mmHg},<15 \mathrm{mmHg}$, $<12 \mathrm{mmHg}$ ) than iStent eyes at 12 months. Given that every $1 \mathrm{mmHg}$ of IOP reduction results in approximately 10\% decreased risk of glaucoma progression [3], the additional $2.4 \mathrm{mmHg}$ of IOP lowering afforded by the iStent inject device is both statistically significant and clinically meaningful.

Coinciding with IOP reduction, both groups achieved clinically and statistically significant reductions in medications from baseline. The percent reduction in medications was significantly greater in iStent inject eyes vs. iStent eyes $(94.1 \%$ vs. $72.2 \%$ reduction, $p=0.023)$. At 12 months postoperative, patients in both groups were able to eliminate an average of 1.6 medications (iStent inject) to 1.3 medications (iStent) from their preoperative regimen. Significantly more iStent inject eyes $(95.7 \%)$ than iStent eyes $(71.4 \%)$ were off medications entirely $(p=0.021)$. The benefits of eliminating medication cannot be overstated, especially for patients who are encumbered by one or multiple topical medications. For example, medications can result in side effects and ocular surface damage $[10,12]$, and they have costs associated with the drops themselves as well as office visits and caregiving needs [43-45]. Additionally, treatment adherence is widely known to be low, which poses a major obstacle to achieving the consistent IOP reduction needed to minimize risk of optic nerve damage and disease progression. Given that adherence markedly declines with multiple rather than single medications, lower medication burden may allow for improved treatment adherence [8]. With respect to this point, it is noteworthy that $95.7 \%$ of iStent inject eyes and $71.4 \%$ of iStent eyes were off medications entirely at 12 months, and no iStent inject eyes and only $14.3 \%$ of iStent eyes were on multiple medications. The fact that IOP also declined over the same time period is further demonstration of the overall benefit of implanting the stents.

The observed IOP and medication benefits were consistently greater with iStent inject versus iStent implantation in this study, confirming the previously observed comparative advantage of iStent inject over iStent in its ability to lower IOP and medications. This advantage is underlain by several key differences in device design between iStent and iStent inject. First, the iStent inject device consists of an injector preloaded with two stents (versus one stent with the iStent device), facilitating up to 6 clock hours of aqueous outflow by bypassing two separate regions of the trabecular meshwork. Each iStent inject stent is designed to carry the $2.5 \mu \mathrm{L} \mathrm{min}^{-1}$ flow of aqueous humor typically produced by the human eye. Second, multidirectional flow and increased access to collector channels are afforded by the four outlet lumens on each iStent inject stent (versus one lumen on the iStent). Third, iStent inject stents are smaller and are implanted more directly into Schlemm's canal than the iStent, promoting more straightforward and uncomplicated surgery and possibly softening the learning curve for surgeons new to the procedure.

The design of both devices is supported by studies in aqueous angiography and computational fluid dynamics (CFD), which is considered the most advanced tool to evaluate stent flow and size. CFD modeling showed that 
although the iStent and iStent inject are microscale devices, each stent had minimal flow resistance and was entirely sufficient to conduct all $2.5 \mu \mathrm{L} \mathrm{min}{ }^{-1}$ of aqueous humor production produced by the human eye [46]. In an in vivo aqueous angiography study, Huang et al. showed significant aqueous outflow improvement after iStent inject implantation with cataract surgery [47]. The study's findings showed that formerly dormant outflow areas could be reactivated, allowing access to up to 6 clock hours of collector channels for aqueous egress.

The use of two versus one stent is supported by preclinical and clinical studies demonstrating enhanced efficacy with two trabecular micro-bypass stents compared to a single stent. Within a whole eye perfusion model, Hunter et al. showed $6.0 \mathrm{mmHg}$ of IOP reduction after implantation of a single iStent, and an additional $2.9 \mathrm{mmHg}$ of IOP reduction after implanting a second iStent, for a total IOP reduction of $8.9 \mathrm{mmHg}$ from baseline [46]. Similarly, using an anterior segment perfusion model, Bahler et al. observed an IOP reduction from 21.4 to $12.4 \mathrm{mmHg}$ after implanting one iStent $(p<0.001)$, and a final IOP of $11.9 \mathrm{mmHg}$ in eyes receiving more than one iStent [48]. Subsequently, Bahler et al. used an anterior segment perfusion model to evaluate the second-generation iStent inject. Results showed that a single iStent inject stent improved outflow facility from 0.16 to $0.38 \mu \mathrm{L} \mathrm{min}^{-1}$ $\mathrm{mmHg}^{-1}(p<0.03, n=7)$, and IOP concurrently decreased from 16.7 to $8.6 \mathrm{mmHg}$; meanwhile, adding a second iStent inject stent further increased outflow facility to $0.78 \mu \mathrm{L} \mathrm{min}^{-1} \mathrm{mmHg}^{-1}(n=2)$ [49].

Three clinical studies corroborated these preclinical findings. Belovay et al. and El Wardani et al. assessed two or three iStents in combination with cataract surgery; and Katz et al. completed a prospective randomized trial comparing outcomes after implanting one, two, or three iStents in standalone surgery $[30,50,51]$. These studies consistently showed that the greatest IOP decrease occurred after placement of a single stent, but that additional stents enabled further IOP reductions. This is possibly due to accessing more clock hours of the distal outflow network and thereby increasing the probability of stenting regions of Schlemm's canal that are patent and functional. Together these six preclinical and clinical studies consistently show that although one stent is responsible for the majority of the reduction in IOP and outflow resistance, additional stents produce incrementally greater reductions.

The safety profile of both groups was excellent, consistent with the substantial evidence base showing the favorable safety of these devices [18-40]. Visual acuity remained stable or improved during follow-up, confirming that stent implantation does not diminish any of the visual benefits expected after cataract surgery. There were no cases of the complications seen with filtering surgeries such as endophthalmitis, choroidal detachment or effusion, hypotony, or bleb-related infections or re-needling [14-16].

Of note, the iStent and iStent inject devices are made of biocompatible titanium that has a proven excellent safety record. Not surprisingly given this biocompatibility, there were no cases of inflammation (e.g., iritis, uveitis) during the entirety of follow-up in either group in this study. Furthermore, only one eye had PAS, which were focal and readily corrected with Nd:YAG laser with no sequelae. This contrasts with the reported PAS rates of up to 30\% observed in trials of other MIGS devices with similar mechanism of action [42, 52-54]. There also were no reports of device dislocation, hypotony, significant hyphema, choroidal detachment, or corneal decompensation such as that cited with some other MIGS devices [55-58].

We acknowledge several limitations in this report. The study was a retrospective, singlesite, single-surgeon case series. All cases were drawn consecutively from the surgeon's heterogeneous patient population and thus were not designed to be identical; however, this real-world design may increase the study's relevance to a broader range of clinical settings and patient populations. The present study had a modest number of eyes in each group and documented outcomes through 12 months postoperative, since the iStent and iStent inject were relatively recently introduced in Brazil; thus, future possibilities for research may be to 
pursue larger sample sizes, longer periods of follow-up, multicenter pooled data, or prospective comparative studies. Since all stent implantations were completed together with cataract surgery, the effect of the stents could not be isolated from that of cataract surgery; however, this simply reflects real-world usage where stents are commonly implanted with cataract surgery. However, since the same surgeon made medication decisions throughout the study, and since medications and IOP are objective numbers, the preoperative values could reasonably be considered as legitimate comparators for postoperative measurements.

\section{CONCLUSIONS}

The present study represents a natural evolution from-and confirmation of-the findings of prior preclinical and clinical studies, with sideby-side data on both iStent and iStent inject in a real-world clinical setting. The findings show clinically and statistically significant IOP and medication reductions and excellent safety after either iStent or iStent inject trabecular microbypass implantation together with cataract surgery. Compared to the iStent group, the iStent inject group achieved significantly greater reductions in IOP and medications from baseline, had significantly lower 12-month mean IOP and medication burden, and had significantly greater proportions of patients achieving lower IOP levels and elimination of medications.

\section{ACKNOWLEDGEMENTS}

We thank the participants of the study.

Funding. No financial sponsorship was received for the work in this study. The Rapid Service Fees were funded by Glaukos Corporation. All authors had full access to all of the data in this study and take complete responsibility for the integrity of the data and accuracy of the data analysis.
Editorial Assistance. Editorial assistance was provided by Glaukos Corporation.

Authorship. All named authors meet the International Committee of Medical Journal Editors (ICMJE) criteria for authorship for this article, take responsibility for the integrity of the work as a whole, and have given their approval for this version to be published.

Disclosures. Dr. Ricardo Guedes is a consultant to Glaukos, Théa France, OftaVision Brasil, and Alcon. Dr. Daniela Marcelo Gravina, Dr. Jonathan Clive Lake, Dr. Vanessa Maria Paletta Guedes, and Dr. Alfredo Chaoubah have nothing to declare.

Compliance with Ethics Guidelines. All procedures performed were in accordance with the ethical standards of the institutional and/or national research committee (the Institutional Review Board (IRB) of the Paletta Guedes Eye Institute), and with the 1964 Helsinki declaration and its later amendments or comparable ethical standards.

Data Availability. The datasets generated during and/or analyzed during the current study are available from the corresponding author on reasonable request.

Open Access. This article is distributed under the terms of the Creative Commons Attribution-NonCommercial 4.0 International License (http://creativecommons.org/licenses/ by-nc/4.0/), which permits any noncommercial use, distribution, and reproduction in any medium, provided you give appropriate credit to the original author(s) and the source, provide a link to the Creative Commons license, and indicate if changes were made.

\section{REFERENCES}

1. Tham YC, Li X, Wong TY, et al. Global prevalence of glaucoma and projections of glaucoma burden through 2040: a systematic review and meta-analysis. Ophthalmology. 2014;121:2081-90. 
2. Leske MC, Heijl A, Hyman L, Bengtsson B, Komaroff E. Factors for progression and glaucoma treatment: the Early Manifest Glaucoma Trial. Curr Opin Ophthalmol. 2004;15:102-6.

3. Heijl A, Leske MC, Bengtsson B, et al. Reduction of intraocular pressure and glaucoma progression: results from the Early Manifest Glaucoma Trial. Arch Ophthalmol. 2002;120:1268-79.

4. Kass MA, Heuer DK, Higginbotham EJ, et al. The Ocular Hypertension Treatment Study: a randomized trial determines that topical ocular hypotensive medication delays or prevents the onset of primary open-angle glaucoma. Arch Ophthalmol. 2002;120:701-13 (discussion 829-830).

5. The AGIS Investigators. The Advanced Glaucoma Intervention Study (AGIS): 7. The relationship between control of intraocular pressure and visual field deterioration. Am J Ophthalmol 2000;130:429-440.

6. American Academy of Ophthalmology Preferred Practice Pattern Glaucoma Panel. Preferred practice pattern: primary open-angle glaucoma. 2015, pp 64-67, 73-74. https://www.aao.org/preferredpractice-pattern/primary-open-angle-glaucomappp-2015. Accessed July 15, 2019.

7. Pillunat LE, Erb C, Jünemann AG, Kimmich F. Micro-invasive glaucoma surgery (MIGS): a review of surgical procedures using stents. Clin Ophthalmol. 2017;11:1583-600.

8. Robin AL, Covert D. Does adjunctive glaucoma therapy affect adherence to the initial primary therapy? Ophthalmology. 2005;112:863-8.

9. Nordstrom BL, Friedman DS, Mozaffari E, Quigley HA, Walker AM. Persistence and adherence with topical glaucoma therapy. Am J Ophthalmol. 2005;140(4):598-606.

10. Baudouin C, Labbé A, Liang H, Pauly A, BrignoleBaudouin F. Preservatives in eyedrops: the good, the bad and the ugly. Prog Retin Eye Res. 2010;29(4):312-34.

11. Newman-Casey PA, Robin AL, Blachley T, et al. The most common barriers to glaucoma medication adherence: a cross-sectional survey. Ophthalmology. 2015;122(7):1308-16.

12. Leung EW, Medeiros FA, Weinreb RN. Prevalence of ocular surface disease in glaucoma patients. J Glaucoma. 2008;17(5):350-5.

13. Glaucoma Laser Trial Research Group. The Glaucoma Laser Trial (GLT) and glaucoma laser trial follow-up study: 7. Results. Am J Ophthalmol. 1995;120:718-31.
14. Rulli E, Biagioli E, Riva I, et al. Efficacy and safety of trabeculectomy vs nonpenetrating surgical procedures: a systematic review and meta-analysis. JAMA Ophthalmol. 2013;131(12):1573-82.

15. Jampel HD, Musch DC, Gillespie BW, et al. Perioperative complications of trabeculectomy in the collaborative initial glaucoma treatment study (CIGTS). Am J Ophthalmol 2005;140(1):16-22.

16. Gedde SJ, Herndon LW, Brandt JD, et al. Postoperative complications in the Tube Versus Trabeculectomy (TVT) study during five years of follow-up. Am J Ophthalmol. 2012;153:804-14.

17. Ahmed IK. MIGS and the FDA: what's in a name? Ophthalmology. 2015;122:1737-9.

18. Samuelson TW, Katz LJ, Wells JM, et al. Randomized evaluation of the trabecular micro-bypass stent with phacoemulsification in patients with glaucoma and cataract. Ophthalmol. 2011;118:459-67.

19. Craven ER, Katz LJ, Wells JM, Giamporcaro JE, iStent Study Group. Cataract surgery with trabecular micro-bypass stent implantation in patients with mild-to-moderate open-angle glaucoma and cataract: two-year follow-up. J Cataract Refract Surg. 2012;38(8):1339-45.

20. Gallardo MJ, Supnet RA, Giamporcaro JE, Hornbeak DM. Outcomes of combined trabecular micro-bypass and phacoemulsification in a predominantly Hispanic patient population. Clin Ophthalmol. 2016;10:1931-7.

21. Neuhann TH. Trabecular micro-bypass stent implantation during small incision cataract surgery for open angle glaucoma or ocular hypertension: long term results. J Cataract Refract Surg. $2015 ; 41: 2664-71$.

22. Ferguson T, Swan R, Ibach M, Schweitzer J, Sudhagoni R, Berdahl JP. Evaluation of a trabecular microbypass stent with cataract extraction in severe primary open-angle glaucoma. J Glaucoma. 2018;27(1):71-6.

23. Ferguson TJ, Swan R, Ibach M, Schweitzer J, Sudhagoni R, Berdahl JP. Trabecular microbypass stent implantation with cataract extraction in pseudoexfoliation glaucoma. J Cataract Refract Surg. 2017;43(5):622-6.

24. Ferguson TJ, Berdakl JP, Schweitzer JA, Sudhagoni RG. Clinical evaluation of a trabecular micro-bypass stent with phacoemulsification in patients with open-angle glaucoma and cataract. Clin Ophthamol. 2016;10:1767-73.

25. Ferguson T, Berdahl J, Schweitzer J, Sudhagoni R. Evaluation of a trabecular micro-bypass stent in 
pseudophakic patients with open-angle glaucoma. J Glaucoma. 2016;25:896-900.

26. Fechtner RD, Voskanyan L, Vold SD, et al. Five-year, prospective, randomized, multi-surgeon trial of two trabecular bypass stents versus prostaglandin for newly-diagnosed open-angle glaucoma. Ophthalmol Glaucoma. 2019;2(3):156-66.

27. Chang DF, Donnenfeld ED, Katz LJ, et al. Efficacy of two trabecular micro-bypass stents combined with topical travoprost in open-angle glaucoma not controlled on two preoperative medications: 3-year follow-up. Clin Ophthalmol. 2017;11:523-8.

28. Arriola-Villalobos P, Martinez-de-la-Casa JM, DiazValle D, et al. Combined iStent trabecular microbypass stent implantation and phacoemulsification for coexisting open-angle glaucoma and cataract: a long-term study. Br J Ophthalmol. 2012;96:645-9.

29. Fea AM, Consolandi G, Zola M, et al. Micro-bypass implantation for primary open-angle glaucoma combined with phacoemulsification: 4-year followup. J Ophthalmol. 2015;26(2015):795357.

30. Katz LJ, Erb C, Carceller Guillamet A, et al. Longterm titrated IOP control with one, two or three trabecular micro-bypass stents in open-angle glaucoma subjects on topical hypotensive medication: 42-month outcomes. Clin Ophthalmol. 2018;12:255-62.

31. Samuelson TW, Sarkisian SR Jr, Lubeck DM, et al. Prospective, randomized, controlled pivotal trial of iStent inject trabecular micro-bypass in primary open-angle glaucoma and cataract: two-year results. Ophthalmology. 2019;126(6):811-21.

32. Hengerer FH, Auffarth GU, Riffel C, Conrad-Hengerer I. Prospective, non-randomized, 36-month study of second-generation trabecular micro-bypass stents with phacoemulsification in various types of glaucoma. Ophthalmol Ther. 2018;7(2):405-15.

33. Hengerer FH, Auffarth GU, Riffel C, Conrad-Hengerer I. Second-generation trabecular micro-bypass stents as standalone treatment for glaucoma: a 36-month prospective study. Adv Ther. 2019;36(7):1606-17.

34. Donnenfeld ED, Solomon KD, Voskanyan L, et al. A prospective 3-year follow-up trial of implantation of two trabecular microbypass stents in open-angle glaucoma. Clin Ophthalmol. 2015;9:2057-65.

35. Fea AM, Belda JI, Rękas M, et al. Prospective unmasked randomized evaluation of the iStent inject $^{\circledR}$ versus two ocular hypotensive agents in patients with primary open-angle glaucoma. Clin Ophthalmol. 2014;8:875-82.
36. Voskanyan L, García-Feijoo J, Belda JI, et al. Prospective, unmasked evaluation of the iStent ${ }^{\circledR}$ inject system for open-angle glaucoma: synergy trial. Adv Ther. 2014;31(2):189-201.

37. Arriola-Villalobos P, Martinez-de-la-Casa JM, DiazValle D, Morales-Fernandez L, Fernandez-Perez C, Garcia-Feijoo J. Glaukos iStent inject ${ }^{\circledR}$ trabecular micro-bypass implantation associated with cataract surgery in patients with coexisting cataract and open-angle glaucoma or ocular hypertension: a long-term study. J Ophthalmol. 2016;2016:1056573.

38. Berdahl J, Voskanyan L, Myers JS, et al. Implantation of two second-generation trabecular microbypass stents and topical travoprost in open-angle glaucoma not controlled on two preoperative medications: 18-month follow-up. Clin Exp Ophthalmol. 2017;45(8):797-802.

39. Lindstrom R, Lewis R, Hornbeak DM, et al. Outcomes following implantation of two second-generation trabecular micro-bypass stents in patients with open-angle glaucoma on one medication: 18-month follow-up. Adv Ther. 2016;33(11):2082-90.

40. Guedes RAP, Gravina DM, Lake JC, Guedes VMP, Chaoubah A. Intermediate results of iStent or iStent inject implantation combined with cataract surgery in a real-world setting: a longitudinal retrospective study. Ophthalmol Ther. 2019;8(1):87-100.

41. Hodapp E, Parrish RK II, Anderson DR. Clinical decisions in glaucoma. St Louis: Mosby; 1993.

42. Samuelson TW, Chang DF, Marquis R, et al. A Schlemm canal microstent for intraocular pressure reduction in primary open-angle glaucoma and cataract: The HORIZON Study. Ophthalmology. 2019;126(1):29-37.

43. Ngan K, Fraser E, Buller S, Buller A. A cost minimisation analysis comparing iStent accompanying cataract surgery and selective laser trabeculoplasty versus topical glaucoma medications in a public healthcare setting in New Zealand. Graefes Arch Clin Exp Ophthalmol. 2018;256(11):2181-9.

44. Patel V, Ahmed I, Podbielski D, Falvey H, Murray J, Goeree R. Cost-effectiveness analysis of standalone trabecular micro-bypass stents in patients with mild-to-moderate open-angle glaucoma in Canada. J Med Econ. 2019;22(4):390-401.

45. Berdahl JP, Khatana AK, Katz LJ, et al. Cost-comparison of two trabecular micro-bypass stents versus selective laser trabeculoplasty or medications only for intraocular pressure control for patients with open-angle glaucoma. J Med Econ. 2017;20(7):760-6. 
46. Hunter K, Fjield T, Heitzmann H, Shandas R, Kahook M. Characterization of micro-invasive trabecular bypass stents by ex vivo perfusion and computational flow modeling. Clin Ophthalmol. 2014;8:499-506.

47. Huang AS, Penteado RC, Papoyan V, Voskanyan L, Weinreb RN. Aqueous angiographic outflow improvement after trabecular micro-bypass in glaucoma patients. Ophthalmol Glaucoma. 2019;2:11-21.

48. Bahler CK, Smedley GT, Zhou J, Johnson DH. Trabecular bypass stents decrease intraocular pressure in cultured human anterior segments. Am J Ophthal. 2004;138(6):988-94.

49. Bahler C, Hann C, Fjield T, Haffner D, Heitzmann $\mathrm{H}$, Fautsch MP. Second-generation trabecular meshwork bypass stent (iStent inject) increases outflow facility in cultured human anterior segments. Am J Ophthal. 2012;153(6):1206-13.

50. Belovay GW, Naqi A, Chan BJ, Rateb M, Ahmed II. Using multiple trabecular micro-bypass stents in cataract patients to treat open-angle glaucoma. J Cataract Refract Surg. 2012;38(11):1911-7.

51. El Wardani M, Bergin C, Achache F, Sharkawi E. Evaluating the trabecular micro-bypass stent combined with phacoemulsification compared to phacoemulsification alone. Klin Monatsbl Augenheilkd. 2015;232:442-5.

52. Pfeiffer N, Garcia-Feijoo J, Martinez-de-la-Casa JM, et al. A randomized trial of a Schlemm's canal microstent with phacoemulsification for reducing intraocular pressure in open angle glaucoma. Ophthalmology. 2015;122:1283-93.

53. Gandolfi SA, Ungaro N, Ghirardini S, et al. Comparison of surgical outcomes between canaloplasty and Schlemm's canal scaffold at 24 months' follow up. J Ophthalmol. 2016;2016:3410469.

54. Fea A. 36 month results from a multicenter randomised study of a Schlemm's canal microstent for reduction of IOP in primary open-angle glaucoma: the HYDRUS II trial. Presented at XXXVI Congress of the European Society of Cataract and Refractive Surgeons (ESCRS); September 22-26, 2018;Vienna, Austria.

55. Gillmann K, Bravetti GE, Mermoud A, Mansouri K. Anterior chamber XEN gel stent movements: the impact on corneal endothelial cell density. J Glaucoma. 2019;28(6):e93-5.

56. Buffault J, Baudouin C, Labbé A. XEN ${ }^{\circledR}$ Gel Stent for management of chronic open angle glaucoma: a review of the literature. J Fr Ophtalmol. 2019;42(2):e37-46.

57. Minckler DS, Baerveldt G, Alfaro MR, Francis BA. Clinical results with the trabectome for treatment of open-angle glaucoma. Ophthalmology. 2005;112(6):962-7.

58. Sieck EG, Epstein RS, Kennedy JB, et al. Outcomes of Kahook Dual Blade goniotomy with and without phacoemulsification cataract extraction. Ophthalmol Glaucoma. 2018;1:75-81. 